\title{
Capsule Commentary on Chan et al., The Effect of a Care Transition Intervention on the Patient Experience of Older, Multi-lingual Adults in the Safety Net: Results of a Randomized Controlled Trial
}

\author{
James F. Burgess, Jr., $P h D^{1,2}$, Eric A. Jones, $M P H^{2}$, Maryum M. Khan, $\mathrm{MPH}^{2}$, and Serena Rajabiun, \\ $M A, M P H^{2}$ \\ ${ }^{1}$ Center for Healthcare Organization and Implementation Research, VA Boston Healthcare System (152M), School of Public Health, Boston \\ University, Boston, MA, USA; ${ }^{2}$ School of Public Health, Boston University, Boston, MA, USA.
}

$\mathrm{J}$ Gen Intern Med 30(12): 1849

DOI: $10.1007 / \mathrm{s} 11606-015-3430-3$

(c) Society of General Internal Medicine 2015

$\mathrm{T}$ he standardized HCAHPS (Hospital Consumer Assessment of Healthcare Providers and Systems) survey does not capture patient- and provider-level contextual factors. This makes the survey susceptible to a range of different interpretations by respondents. ${ }^{1}$ Utilization of patient-centered interventions may improve HCAHPS scores. Chan assesses the impact of receipt of inpatient and post-discharge phone calls by language-concordant providers to reinforce the care plan and to address acute complaints on patient experiences as assessed by HCAHPS.

When discussing any intensive intervention shown not to have a significant impact, it is helpful to think about the context of patient readiness to accept the intervention support. In true person-centered care, the impetus for the intervention would come from a patient's request for support. One factor Chan et al. do not address is social support. ${ }^{2}$ A similar article with a negative intervention result ${ }^{3}$ found that social support may be a key factor in determining patient readiness to accept and use intervention help.

Chan's study suggests a critical aspect for health care systems designing and implementing post-hospitalization programs for elderly populations with low literacy and cognitive impairment. Brief education interventions postdischarge may be insufficient to overcome challenges affecting adherence to care plans once the patient returns home. Involving family and other caregivers in care planning may improve adherence and prevent rehospitalization. For elderly patients with limited social support, care transition programs may also need to move beyond the hospital walls by partnering with community-based agencies to conduct home visits, provide support, and make referrals for necessary medical and psychosocial services.
Randomization, as in Chan's study, is critical, as it can control for potential confounding. However, Chan's study was a negative trial. Findings from such studies often inform local- and national-level policy decisions; thus the import of identification and control of possible confounders is clear and would benefit from a large-scale multi-site study design. ${ }^{4}$ Future randomized controlled trials need to identify and address critical contextual factors such as social support and additional nuances not captured by standardized survey instruments, as this may be essential for the success of interventions delivered to distinct subpopulations of patients.

Acknowledgments: This project was supported in part by grant number T32HSO22242 from the Agency for Healthcare Research and Quality. The content is solely the responsibility of the authors and does not necessarily represent the official views of the Agency for Healthcare Research and Quality. The views expressed in this article are those of the authors and do not necessarily reflect the position or policy of Boston University, the Department of Veterans Affairs, or the United States government.

Conflicts of Interest: The authors have no conflicts of interest with regard to this article.

Corresponding Author: James F. Burgess, Jr., PhD; Center for Healthcare Organization and Implementation Research, VA Boston Healthcare System (152M), School of Public Health, Boston University, 150 South Huntington Avenue, Boston, MA 02130, USA (e-mail: jfburges@bu.edu).

\section{REFERENCES}

1. Jha AK, Orav EJ, Zheng J, Epstein AM. Patients' perception of hospital care in the United States. N Engl J Med. 2008;359:1921-31.

2. Chan B, Goldman LE, Sarkar U, Schneidermann M, Kessell E, Guzman D, Critchfeld J, Kushel M. The effect of a care transition intervention on the patient experience of older, multi-lingual adults in the safety net: results of a randomized controlled trial. J Gen Intern Med. 2015. doi:10.1007/s11606015-3362-y.

3. Altfeld SJ, Shier GE, Rooney M, Johnson TJ, Golden RL, Karavolos K, Avery E, Nandi V, Perry AJ. Effects of an enhanced discharge planning intervention for hospitalized older adults: a randomized trial. The Gerontologist. 2013;53(3):430-40.

4. Weinberger M, Oddone EZ, Henderson WG, Smith DM, Huey J, GiobbieHurder A, Feussner JR. Multisite randomized controlled trials in health services research: scientific challenges and operational issues. Med Care. 2001;39(6):627-34. 